\title{
COMPLETION OF FACTOR ALGEBRAS OF IDEALS
}

\author{
B. BALCAR AND F. FRANEK
}

\begin{abstract}
Let $\Im$ be a $\kappa$-complete ideal over $\kappa$. The structure of the completion of the Boolean algebra $\wp(\kappa) / \Im$ is investigated with respect to properties of the ideal $\Im$ and the cardinal $\kappa$. It is shown that under certain conditions $\operatorname{Comp}(\wp(\kappa) / \Im)$ is isomorphic to a collapse algebra.
\end{abstract}

1. Introduction. In $[\mathbf{B V}]$ it is proven that $\operatorname{Comp}\left(\wp(\kappa) / \Im_{\kappa}\right)$ and $\operatorname{Col}\left(\omega, \kappa^{+}\right)$ are isomorphic if $2^{\kappa}=\kappa^{+}$and $\kappa$ is a regular uncountable cardinal (and where $\Im_{\kappa}$ is the ideal of all subsets of $\kappa$ of size $<\kappa$ ). In this paper we extend this result to:

THEOREM 1. Let $\kappa$ be a regular uncountable cardinal such that $2^{\kappa}=\kappa^{+}$. Let $\Im$ be a $\kappa$-complete nowhere precipitous ideal over $\kappa$. Then $\operatorname{Comp}(\wp(\kappa) / \Im)$ and $\operatorname{Col}\left(\omega, \kappa^{+}\right)$are isomorphic.

Corollary 2. Assume $V=L$. For every regular uncountable cardinal $\kappa$ and every $\kappa$-complete ideal $\Im$ over $\kappa, \operatorname{Comp}(\wp(\kappa) / \Im)$ and $\operatorname{Col}\left(\omega, \kappa^{+}\right)$are isomorphic.

THEOREM 3. Let $\kappa$ be a singular cardinal. Assume $2^{\kappa}=\kappa^{+}$and $2^{\text {cf }(\kappa)}=$ $c f(\kappa)^{+}$.

(1) If $c f(\kappa)=\omega$, then $\operatorname{Comp}(\wp(\kappa) / \Im)$ and $\operatorname{Col}\left(\omega_{1}, \kappa^{+}\right)$are isomorphic.

(2) If $c f(\kappa)>\omega$, then $\operatorname{Comp}\left(\wp(\kappa) / \Im_{\kappa}\right)$ and $\operatorname{Col}\left(\omega, \kappa^{+}\right)$are isomorphic.

REMARK. "If $V=L$, then $\operatorname{Comp}\left(\wp\left(\omega_{1}\right) / \Im\right)$ and $\operatorname{Col}\left(\omega, \omega_{2}\right)$ are isomorphic" is proven independently using a different method in [BTW].

REMARK. Let $\lambda=\mu^{+} \leq \kappa$. Let $\Im$ be a $\lambda$-complete and not $\lambda^{+}$-complete ideal over $\kappa$. Then $\wp(\kappa) / \Im$ as a forcing notion collapses either $\lambda^{+}$to $\lambda$, or $\lambda$ to $\mu$ (see [F]). If $\lambda=\kappa$, the latter happens (see [BTW]).

2. Notation and definitions. Lowercase Greek letters are reserved for ordinals. Let $\kappa, \tau, \mu$ be cardinals.

Let $X, Y$ be sets. $\wp(X)$ is the set of all subsets of $X .{ }^{X} Y$ is the set of all functions from $X$ into $Y$,

$$
\begin{gathered}
{[X]^{<\lambda}=\{Y \subseteq X:|Y|<\lambda\}, \quad[X]^{\lambda}=\{Y \subseteq X:|Y|=\lambda\},} \\
\kappa^{<\lambda}=\sum\left\{\kappa^{\gamma}: \gamma<\lambda \& \gamma \text { is a cardinal }\right\}, \quad<\lambda \kappa=\bigcup\left\{{ }^{\alpha} \kappa: \alpha \in \lambda\right\} .
\end{gathered}
$$

$\operatorname{Col}(\lambda, \kappa)$ is the Boolean completion of the set of all functions $f$ with $\operatorname{dom}(f) \in$ $[\lambda]^{<\lambda}$ and $\operatorname{rng}(f) \subseteq \kappa$ ordered by the inverse inclusion. (In generic extensions obtained via $\operatorname{Col}(\lambda, \kappa)$, the cardinal $\lambda$ is preserved and the cardinal $\kappa$ becomes an ordinal of size $\lambda$.)

Received by the editors March 10, 1986.

1980 Mathematics Subject Classification (1985 Revision). Primary 06E05, 03G05; Secondary 03E05, 04 A20. 
If $X, Y \subset \wp(\kappa)$, then $X \subseteq \subseteq Y$ iff $(\forall y \in Y)(\exists x \in X)(x \subseteq y)$; we say that $X$ refines $Y$, or that $X$ is a refinement of $Y$.

If $B$ is a Boolean algebra, then $1_{B}$ is its greatest element, while $0_{B}$ is its least element. $B^{+}=B-\left\{0_{B}\right\}$. If $b \in B$, then $B \mid b=\{c \in B: c \leq b\}$. Comp $(B)$ denotes the Boolean completion of $B$ (it can be defined as the algebra of regular open sets of the Stone space of $B$ and is unique up to isomorphism).

Let $b \in B^{+} . P \subseteq B^{+}$is a partition of $b$ iff $P$ is a maximal disjoint subfamily of elements of $(B \mid b)^{+}$. If $P_{1}, P_{2}$ are partitions of $b$, then

$$
P_{1} \leq \leq P_{2} \quad \text { iff }\left(\forall c \in P_{1}\right)\left(\exists d \in P_{2}\right)(c \leq d) ;
$$

we say $P_{1}$ refines $P_{2}$, or that $P_{1}$ is a refinement of $P_{2} .\left\langle P_{\alpha}: \alpha<\lambda\right\rangle$ is a descending sequence of partitions of $b$ iff $P_{\alpha} \leq \leq P_{\beta}$ whenever $\beta<\alpha<\lambda$.

$\Im$ is a $\lambda$-complete ideal over $\kappa$ iff $\Im \subseteq \wp(\kappa), \Im \neq \wp(\kappa), \Im$ is closed under unions of size $<\lambda$ and under the subset operation, and $\Im$ contains all singletons of $\kappa$. $\Im^{+}=\wp(\kappa)-\Im . \Im^{*}=\{X \subseteq \kappa:(\kappa-X) \in \Im\}$, which is the dual filter to $\Im$.

For an $A \in \Im^{+}$define $\Im \mid A=\{X \subseteq \kappa:(X \cap A) \in \Im\}$ (which also is a $\lambda$-complete ideal over $\kappa$ and $\Im \subseteq \Im \mid A)$.

$\Im_{\kappa}=\{X \subseteq \kappa:|X|<\kappa\}$ is the Fréchet ideal over $\kappa$.

Let $X, Y \in \Im$. Then $X \subseteq^{*} Y$ iff $(X-Y) \in \Im . P \subseteq \wp(\kappa)$ is $\Im$-disjoint iff $(\forall X \neq Y \in P)(X \cap Y \in \Im) . \quad P \subseteq \wp(\kappa)$ is an almost disjoint family iff $P$ is $\Im_{\kappa}$-disjoint.

Let $S \in \Im^{+}$, then $P \subseteq \wp(S) \cap \Im^{+}$is an $\Im$-partition of $S$ iff $P$ is $\Im$-disjoint and maximal. $\left\langle P_{\alpha}: \alpha \in \lambda\right\rangle$ is a descending sequence of $\Im$-partitions of $S$ iff for every $\alpha \in \lambda, P_{\alpha}$ is an $\Im$-partition of $S$ and $P_{\alpha} \subseteq \subseteq P_{\beta}$ whenever $\alpha<\beta<\lambda$. Let $\left\langle P_{n}: n<\omega\right\rangle$ be a descending sequence of $\Im$-partitions of $S$. Then a sequence $\left\langle X_{n}: n<\omega\right\rangle$ is a path through $\left\langle P_{n}: n<\omega\right\rangle$ iff for every $n \in \omega, X_{n} \in P_{n}$ and also $X_{n+1} \subseteq X_{n}$.

$\Im$ is a precipitous ideal iff for every $S \in \Im^{+}$and for every descending sequence $\left\langle P_{n}: n \in \omega\right\rangle$ of $\Im$-partitions of $S$, there is a path through with a nonempty intersection. $\Im$ is nowhere precipitous iff for every $A \in \Im^{+}, \Im \mid A$ is not precipitous.

Let $X \subseteq \kappa$. Define

$$
\begin{gathered}
X / \Im=\{Y \subseteq \kappa:(X-Y) \cup(Y-X) \in \Im\}, \quad \wp(\kappa) / \Im=\{X / \Im: X \subseteq \kappa\}, \\
X / \Im \wedge Y / \Im=(X \cap Y) / \Im, \quad X / \mid \mathfrak{F} \vee Y / \Im=(X \cup Y) / \Im, \quad-X / \Im=(\kappa-X) / \Im .
\end{gathered}
$$

Thus $\wp(\kappa) / \Im, \wedge$, and $\vee$ form a $\lambda$-complete Boolean algebra (for $\Im$ is $\lambda$-complete) with $\varnothing / \Im=\Im$ being its smallest element and $\kappa / \Im$ being its greatest element. For any $\xi<\lambda$,

$$
\sum\left\{X_{\alpha} / \Im: \alpha<\xi\right\}=\left(\bigcup\left\{X_{\alpha}: \alpha<\xi\right\}\right) / \Im
$$

and

$$
\prod\left\{X_{\alpha}: \alpha<\xi\right\}=\left(\bigcup\left\{X_{\alpha}: \alpha<\xi\right\}\right) / \Im .
$$

Let $\kappa \geq 2$ and $\lambda \geq \omega$. A Boolean algebra $B$ is $(\lambda, \mu, \kappa)$-distributive iff for every sequence $\left\langle P_{\alpha}: \alpha \in \lambda\right\rangle$ of partitions of $1_{B}$ of size $\leq \mu$, here is $Q$, a partition of $1_{B}$, so that $(\forall \alpha \in \lambda)(\forall q \in Q)\left(\left|\left\{p \in P_{\alpha}: p \wedge q \neq 0_{B}\right\}\right|<\kappa\right)$. B is $(\lambda, \cdot, \kappa)$-distributive iff the above holds without the restriction on the size of $P_{\alpha}$ 's. $B$ is $(\lambda, \cdot, \kappa)$-nowhere distributive iff for every $b \in B^{+}, B \mid b$ is not $(\lambda, \cdot, \kappa)$-distributive. 
Let $P$ be a set partially ordered by $\leq . D \subseteq P$ is dense in $P$ iff $(\forall p \in P)(\exists d \in$ $D)(d \leq p) . P$ is $\lambda$-closed iff for any descending sequence $\left\langle p_{\alpha}: \alpha<\beta\right\rangle$ of length $\beta<\lambda$ of elements of $P$, there is a $p \in P$ such that $(\forall \alpha<\beta)\left(p \leq p_{\alpha}\right) \cdot d(P)=$ $\min \{|D|: D$ is dense in $P\}$.

\section{Preliminaries.}

LEMMA 4. Let $\lambda$ be an infinite cardinal and $\kappa$ be a cardinal $\geq 2$. A Boolean algebra $B$ is $(\lambda, \cdot, \kappa)$-nowhere distributive iff there is a sequence $\left\langle P_{\alpha}: \alpha<\lambda\right\rangle$ of partitions of $1_{B}$ such that $\left(\forall b \in B^{+}\right)(\exists \alpha \in \lambda)\left(\left|\left\{p \in P_{\alpha}: p \wedge b \neq 0_{B}\right\}\right| \geq \kappa\right)$.

ProOF. See Lemma 1.11 in [BSV].

LEMMA 5. Let $\lambda$ be an infinite cardinal and $\kappa$ be a cardinal $\geq 2$. Let $B$ be $a$ complete $(\lambda, \cdot, \kappa)$-nowhere distributive Boolean algebra containing a $\lambda$-closed dense subset. Let $d(B)=\kappa^{<\lambda}$. Then $B$ is isomorphic to $\operatorname{Col}(\lambda, \kappa)$.

ProOF. See Theorem 1.15 and Corollary 1.16 in [BSV].

LEMMA 6. Let $\kappa$ be an uncountable cardinal. Let $\Im$ be a countably complete ideal over $\kappa . \Im$ is nowhere precipitous iff there is a descending sequence of $\Im$ partitions of $\kappa$ with no path through whose intersection is nonempty.

ProOF. Assume that $\Im$ is nowhere precipitous (the opposite direction is obvious). Note that if $A \in \Im^{+}$, then $(\Im \mid A)^{+} \subseteq \Im^{+}$and so if $C \in(\Im \mid A)^{+}$and $W$ is an ( $\Im \mid A)$-partition of $C, C \in \Im^{+}$and $W$ is an $\Im$-partition of $C$ as well. Since $\Im$ is nowhere precipitous, $\Im \mid A$ is not precipitous for any $A \in \Im^{+}$. I.e., there are $C \in(\Im \mid A)^{+}$and a descending sequence of $(\Im \mid A)$-partitions with no path through whose intersection is nonempty. Thus there is $C \in \Im^{+}$so that $C \subseteq A$ and

there is a descending sequence $\langle W(C, n): n<\omega\rangle$ of $\Im$-partitions of $C$ with no path through whose intersection is nonempty.

We have just shown that the set of all $C \in \Im^{+}$for which $(*)$ holds is dense in $\left\langle\Im^{+}, \subseteq\right\rangle$. Therefore there is an $\Im$-disjoint partition $F$ of $\kappa$ so that $(*)$ holds for every $C \in F$. Now for every $n<\omega$ define $W_{n}=\bigcup\{W(C, n): C \in F\}$. Since $F$ is an $\Im$-disjoint family, all $W_{n}$ 's are $\Im$-partitions of $\kappa$ and form a descending sequence of partitions. If $\left\langle X_{n}: n<\omega\right\rangle$ is a path through $\left\langle W_{n}: n<\omega\right\rangle$, then $X_{0} \in W(C, 0)$ for some $C \in F$ and since $X_{n} \subseteq X_{0} \subseteq C,\left\langle X_{n}: n<\omega\right\rangle$ is a path through $\langle W(C, n): n\langle\omega\rangle$, and hence its intersection is empty.

LEMMA 7. Let $\kappa, \lambda$ be uncountable cardinals. Let $\Im$ be a $\lambda$-complete nowhere precipitous ideal over $\kappa$. Then $\wp(\kappa) / \Im$ is $\left(\omega, \cdot, \lambda^{+}\right)$-nowhere distributive.

Proof. By Lemma 6 there is a descending sequence $\left\langle W_{n}: N<\omega\right\rangle$ of $\Im$ partitions of $\kappa$ with no path through whose intersection is nonempty. We shall prove that

$$
\left(\forall X \in \Im^{+}\right)(\exists n<\omega)\left(\left|\left\{Y \in W_{n}: X / \Im \wedge Y / \Im \neq \varnothing / \Im\right\}\right| \geq \lambda^{+}\right) .
$$

Then, by Lemma $4, \wp(\kappa) / \Im$ is $\left(\omega, \cdot, \lambda^{+}\right)$-nowhere distributive. By way of contradiction assume $\left(\exists X \in \Im^{+}\right)(\forall n<\omega)\left(\left|\left\{Y \in W_{n}: X / \Im \wedge Y / \Im\right\}\right|<\lambda^{+}\right)$. Since $W_{n}$ is an $\Im$-partition of $\kappa, Q_{n}=\left\{Y / \Im: Y \in W_{n} \& X / \Im \wedge Y / \Im \neq \varnothing / \Im\right\}$ is a partition of $X / \Im$ of size $\leq \lambda$ (in the Boolean algebra $\wp(\kappa) / \Im$ ). For each $Y / \Im \in Q_{n}$ 
choose a representative and enumerate them, i.e. $Q_{n}=\left\{Y_{\alpha}^{n} / \Im: \alpha<\lambda_{n}\right\}$, where $\lambda_{n}=\left|Q_{n}\right| \leq \lambda$. Let $\hat{Q}_{n}=\left\{Y_{\alpha}^{n}: \alpha<\lambda_{n}\right\}$. Then $\hat{Q}_{n}$ is an $\Im$-partition of $X$ of size $\lambda_{n}$. Define $Z_{0}^{n}=Y_{0}^{n}$ and $Z_{\alpha}^{n}=Y_{\alpha}^{n}-\bigcup\left\{Y_{\beta}^{n}: \beta<\alpha\right\}$ for all $\alpha<\lambda_{n}$. Since $\Im$ is $\lambda$-complete and $\lambda_{n} \leq \lambda,\left\{Z_{\alpha}^{n}: \alpha<\lambda_{n}\right\}$ is a disjoint $\Im$-partition of $X$ refining $\hat{Q}_{n}$. Let $Z_{n}=X-\bigcup\left\{Z_{\alpha}^{n}: \alpha<\lambda_{n}\right\}$. Then every $Z_{n} \in \Im$ and so $X-\bigcup\left\{Z_{n}: n<\omega\right\} \neq \varnothing$, for $\Im$ is countable complete. Pick any $\rho \in X-\bigcup\left\{Z_{n}: n<\omega\right\}$. For every $n<\omega$, $\rho \in X$ and $\rho \notin Z_{n}$, hence there must be some $\alpha_{n}<\lambda_{n}$ so that $\rho \in Z_{\alpha_{n}}^{n} \subseteq Y_{\alpha_{n}}^{n}$. Since $\left\langle W_{n}: n<\omega\right\rangle$ is a descending sequence of $\Im$-partitions of $\kappa,\left\langle Y_{\alpha_{n}}^{n}: n<\omega\right\rangle$ is a (descending) path through $\left\langle W_{n}: n<\omega\right\rangle$ which has a nonempty intersection (for it contains at least $\rho$ ), a contradiction.

LEMMA 8. Let $\kappa, \lambda$ be cardinals, $\kappa \geq 2$ and $\lambda \geq \omega$. Let $B$ be $a(\lambda, \cdot, \kappa)$-nowhere distributive Boolean algebra. Then

(1) $B$ is atomless and thus $\omega$-closed,

(2) if $D \subseteq B$ is dense in $B$, then $|D| \geq \kappa$,

(3) $\operatorname{Comp}(B)$ is also $(\lambda, \cdot, \kappa)$-nowhere distributive.

PROOF. Easy, left to the interested reader.

LEMMA 9. Let $\kappa, \lambda$ be uncountable cardinals. Let $\Im$ be a $\lambda$-complete nowhere precipitous ideal over $\kappa$ so that $\wp(\kappa) / \Im$ has a dense set of size $\lambda^{+}$. Then $\operatorname{Comp}(\wp(\kappa) / \Im)$ and $\operatorname{Col}\left(\omega, \lambda^{+}\right)$are isomorphic.

PROOF. Since $\Im$ is $\lambda$-complete and nowhere precipitous, by Lemma $6, \wp(\kappa) / \Im$ is $\left(\omega, \cdot, \lambda^{+}\right)$-nowhere distributive. By Lemma $8, \operatorname{Comp}(\wp(\kappa) / \Im)$ is also $\left(\omega, \cdot, \lambda^{+}\right)$nowhere distributive and $d(\operatorname{Comp}(\wp(\kappa) / \Im)) \geq \lambda^{+}$. Since $\wp(\kappa) / \Im$ has a dense set of size $\lambda^{+}$, so does $\operatorname{Comp}(\wp(\kappa) / \Im)$. Hence $d(\operatorname{Comp}(\wp(\kappa) / \Im))=\lambda^{+}=\left(\lambda^{+}\right)^{<\omega}$. By Lemma $8, \wp(\kappa) / \Im$ is $\omega$-closed. Thus all requirements of Lemma 5 are satisfied and so $\operatorname{Comp}(\wp(\kappa) / \Im)$ and $\operatorname{Col}\left(\omega, \lambda^{+}\right)$are isomorphic.

These were preliminaries needed to deal with ideals over regular cardinals. Let us now turn our attention to singular cardinals. Unfortunately, we can only describe the structure of $\operatorname{Comp}\left(\wp(\kappa) / \Im_{\kappa}\right)$.

Let $\omega \leq \lambda=c f(\kappa)<\kappa$. (Recall that $\lambda$ is regular, $\wp(\lambda) / \Im_{\lambda}$ can be regularly imbedded into $\wp(\kappa) / \Im_{\kappa}$, and $\Im_{\kappa}$ is $\lambda$-complete.)

As usual, for $X, Y \in[\lambda]^{\lambda}$ define $X \subseteq^{*} Y$ iff $(X-Y) \in \Im_{\lambda}$, i.e. iff $|X-Y|<\lambda$.

LEMMA 10. Let $\lambda \geq \omega$ be regular. For every $X \in[\lambda]^{\lambda}$ define by induction $h_{X}(\alpha)=\min \left(\lambda-\bigcup\left\{h_{X}(\beta): \beta<\alpha\right\}\right)$ for all $\alpha \in X$. Then

(1) $h_{X}$ is strictly increasing,

(2) $(\forall \alpha \in X)\left(h_{X}(\alpha) \leq \alpha\right)$,

(3) if $Y \in[\lambda]^{\lambda}$ and $Y \subseteq \subseteq^{*} X$, then

$$
(\exists \beta \in X \cap Y)(\forall \alpha \in X \cap Y)\left(\alpha \geq \beta \rightarrow h_{X}(\alpha) \geq h_{Y}(\alpha)\right),
$$

(4) if $s: X \rightarrow \lambda$ is strictly increasing, then $(\forall \alpha \in X)\left(h_{X}(\alpha) \leq s(\alpha)\right)$.

Proof. Standard, left to the reader. (Note that $h_{X}$ is the inverse of the enumeration function for $X$.)

LEMMA 11. Let $\omega \leq \lambda=c f(\kappa)<\kappa$. Assume that there is a sequence $\left\langle P_{\alpha}: \alpha \in \mu\right\rangle$ of $\Im_{\lambda}$-partitions of $\lambda$ so that $\left(\forall X \in[\lambda]^{\lambda}\right)(\exists \alpha \in \mu)\left(\exists Y \in P_{\alpha}\right)\left(Y \subseteq^{*} X\right)$. Then $\wp(\kappa) / \Im_{\kappa}$ is $\left(\mu, \cdot \kappa^{+}\right)$-nowhere distributive. 
ProOF. Let $\kappa=\bigcup\left\{Q_{\alpha}: \alpha \in \lambda\right\}$ where $\left\langle Q_{\alpha}: \alpha \in \lambda\right\rangle$ is a disjoint family of subsets of $\kappa$ so that $\left\langle\left|Q_{\alpha}\right|: \alpha \in \lambda\right\rangle$ is a strictly increasing sequence of regular cardinals and $\kappa=\sum\left\{\left|Q_{\alpha}\right|: \alpha \in \lambda\right\}$. Let $\kappa_{\alpha}=\left|Q_{\alpha}\right|$ for every $\alpha \in \lambda$. For any $X \in[\lambda]^{\lambda}$ define $\hat{X}=\bigcup\left\{Q_{\alpha}: \alpha \in X\right\}$. Let $h_{X}: X \rightarrow \lambda$ be as in Lemma 10. Define

$S_{X}=\left\{D \in[\kappa]^{\kappa}:(\forall \alpha \in X)\left(\left|D \cap Q_{\alpha}\right| \leq\left|Q_{h_{X}(\alpha)}\right|\right) \&(\forall \alpha \in(\lambda-X))\left(D \cap Q_{\alpha}=\varnothing\right)\right\}$.

Claim 1. For any $X \in[\lambda]^{\lambda}, S_{X}$ is dense in $\left([\hat{X}]^{\kappa}, \subseteq\right)$.

If $D \in S_{X}$, then $D=\bigcup\left\{D \cap Q_{\alpha}: \alpha \in X\right\} \subseteq \bigcup\left\{Q_{\alpha}: \alpha \in X\right\}=\hat{X}$, so $D \in[\hat{X}]^{\kappa}$. Let $Y \in[\hat{X}]^{\kappa}$. Then

$$
(\forall \gamma \in \lambda)(\forall \beta \in X)(\exists \alpha \in X)\left(\alpha>\beta \&\left|Y \cap Q_{\alpha}\right| \geq\left|Q_{\gamma}\right|\right) .
$$

If not, then $(\exists \gamma \in \lambda)(\exists \beta \in X)(\forall \alpha \in X)\left(\alpha>\beta \rightarrow\left|Y \cap Q_{\alpha}\right|<\left|Q_{\gamma}\right|\right)$ and so $|Y|<\left|Q_{\beta}\right|+\left|G_{\gamma}\right|<\kappa$, a contradiction. Thus we can define by induction a strictly increasing function $f: \lambda \rightarrow X$ so that $\left|Y \cap Q_{f(\gamma)}\right| \geq\left|Q_{\gamma}\right|$ for all $\gamma \in \lambda$ and $\gamma \leq h_{X}(f(\gamma)) \leq f(\gamma)$. For every $\alpha \in X$ define $D_{\alpha}$ by

(i) if $\alpha=f(\gamma)$ for some $\gamma \in \lambda$, then let $D_{\alpha}$ be a subset of $\left(Y \cap Q_{f(\gamma)}\right)$ of size $\geq\left|Q_{\gamma}\right|$ but $\leq\left|Q_{h_{X}(f(\gamma))}\right|$. Notice that this is possible as $\gamma \leq h_{X}(f(\gamma))$ and so $\left|Q_{\gamma}\right| \leq\left|Q_{h_{X}(f(\gamma))}\right|$,

(ii) otherwise $D_{\alpha}=\varnothing$.

Let $D=\bigcup\left\{D_{\alpha}: \alpha \in X\right\}$. Then $D \subseteq Y$, and $|D| \geq \sum\left\{\left|Q_{\gamma}\right|: \gamma \in\right.$ $\operatorname{rng}(f)\}=\kappa$ as $\operatorname{rng}(f)=\lambda$, and also $\left|D \cap Q_{\alpha}\right| \leq\left|Q_{h_{X}(\alpha)}\right|$ for all $\alpha \in X$. Hence $D \in S_{X}$ and so the claim is proven.

By Claim 1, for any $X \in[\lambda]^{\lambda}$ there is an almost disjoint partition of $\hat{X}$ consisting entirely of elements of $S_{X}$. Choose one and denote it $R_{X}$. Define

$$
W_{\alpha}=\bigcup\left\{R_{X}: X \in P_{\alpha}\right\}
$$

for every $\alpha \in \mu$.

Claim 2. Each $W_{\alpha}$ is an almost disjoint partition of $\kappa$.

Clearly, for each $Z \in W_{\alpha},|Z|=\kappa$. Now let $Z_{1}, Z_{2} \in W_{\alpha}$. If $Z_{1}, Z_{2} \in R_{X}$ for some $X \in P_{\alpha}$, then $\left|Z_{1} \cap Z_{2}\right|<\kappa$. So assume that $Z_{1} \in R_{X_{1}}, Z_{2} \in R_{X_{2}}$, and $X_{1} \neq X_{2} \in P_{\alpha}$. Assume by way of contradiction that $\left|Z_{1} \cap Z_{2}\right|=\kappa$. If $\beta \in Z_{1} \cap Z_{2}$, then $\beta \in \hat{X}_{1} \cap \hat{X}_{2}$, so $\beta \in Q_{\gamma}$ for some $\gamma \in X_{1} \cap X_{2}$. Thus there is a function $f:\left(Z_{1} \cap Z_{2}\right) \rightarrow\left(X_{1} \cap X_{2}\right)$ so that $\beta \in Q_{f(\beta)}$. Since $\left|X_{1} \cap X_{2}\right|<\lambda$, and hence $|\operatorname{rng}(f)|<\lambda$, there are $\gamma \in X_{1} \cap X_{2}$ and $Z_{3} \in\left[Z_{1} \cap Z_{2}\right]^{\kappa}$ such that $f(\beta)=\gamma$ for all $\beta \in Z_{3}$. Then $Z_{3} \subseteq Q_{\gamma}$, so $\left|Q_{\gamma}\right|=\kappa$, a contradiction. Hence $\left|Z_{1} \cap Z_{2}\right|<\kappa$. The claim is proven.

Claim 3. $\left\{W_{\alpha}: \alpha \in \mu\right\}$ witnesses that $\wp(\kappa) / \Im_{\kappa}$ is $\left(\mu, \cdot, \kappa^{+}\right)$-nowhere distributive.

Let $C \in[\kappa]^{\kappa}$. Then $(\forall \gamma \in \lambda)(\forall \beta \in \lambda)(\exists \alpha \in \lambda)(\alpha>\beta \& \mid C \cap$ $\left.Q_{\alpha}|>| Q_{\gamma} \mid\right)$. If not, then $(\exists \gamma \in \lambda)(\exists \beta \in \lambda)(\forall \alpha \in \lambda)(\alpha>\beta \rightarrow$ $\left.\left|C \cap Q_{\alpha}\right| \leq\left|Q_{\gamma}\right|\right)$. Then $|C| \leq\left|Q_{\beta}\right|+\left|Q_{\gamma}\right|<\kappa$, a contradiction. Thus there is a strictly increasing sequence $\left\langle\alpha_{\gamma}: \gamma \in \lambda\right\rangle \subseteq \lambda$ so that $\left|C \cap Q_{\alpha_{\gamma}}\right|>\left|Q_{\gamma}\right|$. Let $X=\left\{\alpha_{\gamma}: \gamma \in \lambda\right\}$. So $X \in[\lambda]^{\lambda}$. Define $s\left(\alpha_{\gamma}\right)=\gamma$ for all $\gamma \in \lambda$. Thus $s: X \rightarrow \lambda$ is strictly increasing and 
therefore $h_{X}(\alpha) \leq s(\alpha)$ for all $\alpha \in X$. By the assumption of this lemma, $(\exists \delta \in \mu)\left(\exists Y \in P_{\delta}\right)\left(Y \subseteq^{*} X\right)$. Then there is $\bar{\alpha} \in X \cap Y$ so that $h_{Y}(\alpha) \leq h_{X}(\alpha)$ for all $\alpha \in(X \cap Y)-\bar{\alpha}$. Recall that $R_{Y}$ is an almost disjoint partition of $\hat{Y}$ consisting of elements of $S_{Y}$, and also $R_{Y} \subseteq W_{\delta}$. If $Z \in R_{Y}$, then $Z \in[\kappa]^{\kappa},(\forall \alpha \in Y)\left(\left|Z \cap Q_{\alpha}\right| \leq\right.$ $\left.\left|Q_{h_{Y}(\alpha)}\right|\right)$ and $(\forall \alpha \in \lambda-Y)\left(Z \cap Q_{\alpha}=\varnothing\right)$.

There are at least $\kappa^{+}$elements of $R_{Y}$ which have an intersection of size $\kappa$ with $C$.

If not, let $\left\{Z_{\rho}: \rho \in \kappa\right\}$ be a list of elements of $R_{Y}$ which contains all $Z \in R_{Y}$ so that $|Z \cap C|=\kappa$ (if need be, with repetitions). Let $D=C \cap \hat{X} \cap \hat{Y}$. Then

$(\forall \beta \in \lambda)(\exists \gamma \in \lambda)\left(\gamma>\beta \&\left|\left(D-\bigcup\left\{Z_{\rho}: \rho \in \kappa_{\beta}\right\}\right) \cap Q_{\alpha_{\gamma}}\right|>\left|Q_{\gamma}\right|\right)$.

Let $\beta \in \lambda$. Since $Y \subseteq^{*} X,|X \cap Y|=\lambda$. There is $\gamma \in \lambda$ so that $\gamma>\beta$ and $\alpha_{\gamma} \in(X \cap Y)-\bar{\alpha}$. If $\rho<\kappa_{\beta}$, then $\left|Z_{\rho} \cap Q_{\alpha_{\gamma}}\right| \leq$ $\left|Q_{h_{Y}\left(\alpha_{\gamma}\right)}\right| \leq\left|Q_{h_{X}\left(\alpha_{\gamma}\right)}\right| \leq\left|Q_{s\left(\alpha_{\gamma}\right)}\right|=\left|Q_{\gamma}\right|$. Thus

$$
\left|\bigcup\left\{Z_{\rho}: \rho<\kappa_{\beta}\right\} \cap Q_{\alpha_{\gamma}}\right| \leq \kappa_{\beta} \times\left|Q_{\gamma}\right|=\left|Q_{\gamma}\right|
$$

$$
\text { as } \gamma>\beta \text { and }\left|Q_{\gamma}\right|=\kappa_{\gamma} .
$$

Since $\alpha_{\gamma} \in X \cap Y, Q_{\alpha_{\gamma}} \subseteq \hat{X} \cap \hat{Y} .\left|D \cap Q_{\alpha_{\gamma}}\right|=\left|(C \cap \hat{X} \cap \hat{Y}) \cap Q_{\alpha_{\gamma}}\right|=$ $\left|C \cap Q_{\alpha_{\gamma}}\right|>\left|Q_{\gamma}\right|$. Thus

$$
\begin{aligned}
\mid(D & \left.-\bigcup\left\{Z_{\rho}: \rho \in \kappa_{\beta}\right\}\right) \cap Q_{\alpha_{\gamma}} \mid \\
& =\left|\left(D \cap Q_{\alpha_{\gamma}}\right)-\left(\bigcup\left\{Z_{\rho}: \rho<\kappa_{\beta}\right\} \cap Q_{\alpha_{\gamma}}\right)\right|>\left|Q_{\gamma}\right| .
\end{aligned}
$$

Therefore $(* *)$ holds.

By $(* *)$ there is a strictly increasing sequence $\left\langle\gamma_{\beta}: \beta\langle\lambda\rangle \subseteq \lambda\right.$ so that $\left|\left(D-\bigcup\left\{Z_{\rho}: \rho \in \kappa_{\beta}\right\}\right) \cap Q_{\alpha_{\gamma \beta}}\right|>\left|Q_{\gamma \beta}\right|$. For each $\beta \in \lambda$ let $A_{\beta}$ be a subset of $\left(D-\bigcup\left\{Z_{\rho}: \rho \in \kappa_{\beta}\right\}\right) \cap Q_{\alpha_{\gamma \beta}}$ of size $\left|Q_{\gamma \beta}\right|$. Let $A=\bigcup\left\{A_{\beta}: \beta \in \lambda\right\}$. Then $A \subseteq D$, so $A \subseteq \hat{Y}$ and $A \subseteq C$. $|A|=$ $\sum\left\{\left|A_{\beta}\right|: \beta \in \lambda\right\}=\sum\left\{\left|Q_{\gamma \beta}\right|: \beta \in \lambda\right\}=\sum\left\{\kappa_{\gamma \beta}: \beta \in \lambda\right\}=\kappa$. So $A \in|\hat{Y}|^{\kappa}$. Since $R_{Y}$ is an almost disjoint partition of $\hat{Y}$, for some $Z \in R_{Y},|Z \cap A|=\kappa$. Then also $|Z \cap C|=\kappa$. Therefore $Z=Z_{\rho_{0}}$ for some $\rho_{0} \in \kappa$. Let $\beta \in \lambda$ so that $\rho_{0}<\kappa_{\beta}$ and $Z \cap A_{\beta} \neq \varnothing$. Let $x \in Z \cap A_{\beta}$. Then $x \notin \bigcup\left\{Z_{\rho}: \rho<\kappa_{\beta}\right\}$, so $x \notin Z_{\rho_{0}}=Z$, a contradiction. Hence $(*)$ holds.

Thus Claim 3 is proven.

This completes the proof of the lemma.

LEMMA 12. Let $\lambda \geq \omega_{1}$ be regular. Let $B=\operatorname{Col}\left(\omega, \lambda^{+}\right)$. There is a descending sequence $\left\langle P_{n}: n<\omega\right\rangle$ of partitions of $1_{B}$ such that $\left(\forall b \in B^{+}\right)(\exists n<\omega)(\exists c \in$ $\left.P_{n}\right)(c<b)$ and so that $\bigcup\left\{P_{n}: n<\omega\right\}$ is dense in $B$.

Proof [SKetch]. Let $C=<\omega\left(\lambda^{+}\right)$. For $f, g \in C$ let $f \preceq g$ iff $g \subseteq f$. Then $B=\operatorname{Comp}((C, \preceq))$. For every $n<\omega$, let $P_{n}={ }^{n}\left(\lambda^{+}\right)$. Now it is easy to check that $\left\langle P_{n}: n<\omega\right\rangle$ is a required sequence. 
LEMMA 13. Let $2^{\omega}=\omega_{1}$. There is a sequence $\left\langle P_{\alpha}: \alpha \in \omega_{1}\right\rangle$ of $\Im_{\omega}$-partitions of $\omega$ such that $\left(\forall X \in[\omega]^{\omega}\right)\left(\exists \alpha \in \omega_{1}\right)\left(\exists Y \in P_{\alpha}\right)(Y \subseteq X)$.

PROOF. Easy, left to the reader (or follows from Base Matrix Theorem in [BPS] $($ see $[\mathrm{BVo}]))$.

Lemma 14. Let $\omega=c f(\kappa)<\kappa$. Then $\wp(\kappa) / \Im_{\kappa}$ is $\omega_{1}$-closed.

PROOF. Let $\left\langle\kappa_{n}: n\langle\omega\rangle\right.$ be an increasing sequence of regular cardinals cofinal in $\kappa . \wp(\kappa) / \Im_{\kappa}$ is atomless, for if $X \notin \Im_{\kappa}$, then $|X|=\kappa$ and so there is $Y \subseteq X$ so that $|Y|=\kappa$ and $|X-Y|=\kappa$, hence $\varnothing / \Im_{\kappa}<Y / \Im_{\kappa}<X / \Im_{\kappa}$ and so $X / \Im_{\kappa}$ is not an atom. Thus $\wp(\kappa) / \Im_{\kappa}$ is $\omega$-closed. Let $\left\langle X_{n} / \Im_{\kappa}: n<\omega\right\rangle$ be a strictly decreasing sequence of elements of $\wp(\kappa) / \Im_{\kappa}$. Let $Y_{n}=\bigcap\left\{X_{m}: m \leq n\right\}$ for all $n<\omega$. Since $X_{n}=Y_{n} \cup \bigcup\left\{X_{n}-X_{m}: m<n\right\}$ and $\bigcup\left\{X_{n}-X_{m}: m<n\right\} \in \Im_{\kappa}, Y_{n} / \Im_{\kappa}=$ $X_{n} / \Im_{\kappa}$ for every $n<\omega$. Thus $\left\langle Y_{n} / \Im_{\kappa}: n<\omega\right\}$ is a strictly decreasing sequence of elements of $\wp(\kappa) / \Im_{\kappa}$, and therefore $\left|Y_{n+1}-Y_{n}\right|=\kappa$ for all $n<\omega$. For each $n<\omega$ choose some $A_{n} \subset\left(Y_{n}-Y_{n+1}\right)$ of size $\kappa_{n}$. Let $A=\bigcup\left\{A_{n}: n<\omega\right\}$. Then $|A|=\sum\left\{\kappa_{n}: n<\omega\right\}=\kappa, A \subseteq Y_{0}$, and for every $n \geq 1, A-Y_{n}=\bigcup\left\{A_{i}: i<n\right\}$, hence $A \subseteq \subseteq^{*} Y_{n}$ and so $A \subseteq^{*} X_{n}$ for all $n<\omega$. Thus $\varnothing / \Im_{\kappa}<A / \Im_{\kappa} \leq X_{n} / \Im_{\kappa}$ for all $n<\omega$.

\section{Main results.}

ProOF OF THEOREM 1. By Lemma $7, \wp(\kappa) / \Im$ is $\left(\omega, \cdot, \kappa^{+}\right)$-nowhere distributive and so by Lemma $8 d(\wp(\kappa) / \Im) \geq \kappa^{+}$. Since $|\wp(\kappa) / \Im| \leq|\wp(\kappa)|=2^{\kappa}=\kappa^{+}$, $d(\wp(\kappa / \Im))=\kappa^{+}$and so $\wp(\kappa) / \Im$ has a dense set of size $\kappa^{+}$. Thus, by Lemma 9 , $\operatorname{Comp}(\wp(\kappa) / \Im)$ and $\operatorname{Col}\left(\omega, \kappa^{+}\right)$are isomorphic.

Proof OF COROLlaRY 2. It is known that under $V=L$, there are no measurable cardinals, and hence no countably complete precipitous ideals (see e.g. $[\mathbf{J}]$ ) and that the G.C.H. holds. Therefore for any $\kappa$-complete ideal $\Im$ over $\kappa$, all requirements of Theorem 1 are satisfied, and so $\operatorname{Comp}(\wp(\kappa) / \Im)$ and $\operatorname{Col}\left(\omega, \kappa^{+}\right)$are isomorphic.

NOTE. Why cannot Theorem 1 be applied to singular cardinals? For if $\Im$ is a $\kappa$-complete ideal over a singular cardinal $\kappa$, then $\kappa \in \Im$ and it is a contradiction. Is it necessary that $\Im$ be $\kappa$-complete? As long as we can find a dense set of size $\lambda^{+}$in $\wp(\kappa) / \Im$, we need just $\lambda$-completeness (see Lemma 9 ). Thus we need $\kappa$-completeness only to get "close" to the "natural" estimate of the size of $\wp(\kappa) / \Im$.

Proof of Theorem 3. Let $\kappa$ be a singular cardinal. Assume $2^{\kappa}=\kappa^{+}$and $2^{c f(\kappa)}=c f(\kappa)^{+}$.

(1) Assume that $c f(\kappa)=\omega$.

By Lemmas 13 and 11, $\wp(\kappa) / \Im_{\kappa}$ is $\left(\omega_{1}, \cdot, \kappa^{+}\right)$-nowhere distributive. By Lemma 8 , $\operatorname{Comp}\left(\wp(\kappa) / \Im_{\kappa}\right)$ is $\left(\omega_{1}, \cdot, \kappa^{+}\right)$-nowhere distributive. By Lemma $14, \wp(\kappa) / \Im_{\kappa}$ is $\omega_{1}$-closed. By Lemma $8, d\left(\operatorname{Comp}\left(\wp(\kappa) / \Im_{\kappa}\right)\right) \geq \kappa^{+}$. Since $\left|\wp(\kappa) / \Im_{\kappa}\right| \leq 2^{\kappa}=$ $\kappa^{+}, d\left(\operatorname{Comp}\left(\wp(\kappa) / \Im_{\kappa}\right)\right)=\kappa^{+}$. Since $2^{\kappa}=\kappa^{+}$and $c f(\kappa)=\omega,\left(\kappa^{+}\right)^{<\omega_{1}}=\kappa^{+}$. Therefore, by Lemma $5, \operatorname{Comp}\left(\wp(\kappa) / \Im_{\kappa}\right)$ and $\operatorname{Col}\left(\omega_{1}, \kappa^{+}\right)$are isomorphic.

(2) Assume that $\lambda=c f(\kappa)>\omega$.

By Theorem 1, $\operatorname{Comp}\left(\wp(\lambda) / \Im_{\lambda}\right)$ is isomorphic to $\operatorname{Col}\left(\omega, \lambda^{+}\right)$, for $\Im_{\lambda}$ is nowhere precipitous (see $[\mathbf{J}]$ or $[\mathbf{J P}]$ ), $\lambda$-complete and $2^{\lambda}=\lambda^{+}$. Thus, by Lemma 12 , 
there is a descending sequence $\left\langle P_{n}: n<\omega\right\rangle$ of $\Im_{\lambda}$-partitions of $\lambda$ so that $(\forall X \in$ $\left.[\lambda]^{\lambda}\right)(\exists n<\omega)\left(\exists Y \in P_{n}\right)\left(Y \subseteq^{*} X\right)$. Hence, by Lemma 11, $(\kappa) / \Im_{\kappa}$ is $\left(\omega, \cdot, \kappa^{+}\right)$nowhere distributive. By Lemma $8, \operatorname{Comp}\left(\wp(\kappa) / \Im_{\kappa}\right)$ is $\left(\omega, \cdot, \kappa^{+}\right)$-nowhere distributive, $d\left(\operatorname{Comp}\left(\wp(\kappa) / \Im_{\kappa}\right)\right) \geq \kappa^{+}$, and $\wp(\kappa) / \Im_{\kappa}$ is $\omega$-closed. Since $\left|\wp(\kappa) / \Im_{\kappa}\right| \leq$ $2^{\kappa}=\kappa^{+}, d\left(\operatorname{Comp}\left(\wp(\kappa) / \Im_{\kappa}\right)\right)=\kappa^{+}$. Clearly $\left(\kappa^{+}\right)^{<\omega}=\kappa^{+}$and so, by Lemma 5 , $\operatorname{Comp}\left(\wp(\kappa) / \Im_{\kappa}\right)$ and $\operatorname{Col}\left(\omega, \kappa^{+}\right)$are isomorphic.

\section{REFERENCES}

[BPS] B. Balcar, J. Pelant and P. Simon, The space of ultrafitters on $N$ covered by nowhere dense sets, Fund. Math. 110 (1980), 11-24.

[BSV] B. Balcar, P. Simon and P. Vojtás, Refinement properties and extensions of filters in Boolean algebras, Trans. Amer. Math. Soc. 267 (1981), 265-283.

[BTW] J. E. Baumgartner, A. D. Taylor and S. Wagon, Ideals on uncountable cardinals, Logic Colloquium 77, Proc. Conf. Wroclaw, 1977.

[BV] B. Balcar and P. Vopěnka, On systems of almost disjoint sets, Bull. Acad. Polon. Sci. Sér. Sci. Tech. 20 (1972).

[BVo] B. Balcar and P. Vojtáś, Almost disjoint refinement of families of subsets of $N$, Proc. Amer. Math. Soc. 79 (1980), 465-470.

[F] F. Franek, Some results about saturated ideals and about isomorphisms of $\kappa$-trees, Ph.D. Dissertation, Dept. of Math., University of Toronto, 1983.

[J] T. Jech, Set theory, Academic Press, 1978.

[JP] T. Jech and K. Prikry, Ideals over uncountable sets: applications of almost disjoint functions and generic ultrapowers, Mem. Amer. Math. Soc., No. 214 (1979).

ČKD Praha O.P. závod Polovodiče, 14000 Praha 4, Czechoslovakia

Department of COMPUTER SCIENCE AND Systems, MCMaster University, HAMILTON, ONTARIO, CANADA L8S 4K1 